\title{
Suboptimal use of gastroprotective medication in elderly antiplatelet users
}

\author{
SlezakovaV ${ }^{1}$, Varga $\mathrm{Z}^{1}$, Murin $\mathrm{J}^{2}$, Schweigertova $\mathrm{J}^{3}$, Jankyova $\mathrm{S}^{3}$, Foltanova $\mathrm{T}^{3}$, Zabka $\mathrm{M}^{4}$, \\ Bozik $\mathrm{M}^{5}$, Wawruch $\mathrm{M}^{1}$
}

Department of Pharmacology and Clinical Pharmacology, Faculty of Medicine, Comenius University in Bratislava, Bratislava, Slovakia. veronika.slezakova@fmed.uniba.sk

\begin{abstract}
Background: Use of acetylsalicylic acid (ASA) or thienopyridines in monotherapy or combination of both drugs is associated with increased risk of gastrointestinal (GI) bleeding. The administration of drugs inhibiting gastric acid production represents an effective way to avoid GI disorders associated with antiplatelet therapy. Objectives: The aim of our study was to evaluate the use of gastroprotective medication in elderly antiplatelet users in relation to risk factors for GI bleeding.

Methods: Patients $(n=428)$ aged $\geq 65$ years who were prescribed low dose ASA or clopidogrel in monotherapy or combination at hospital discharge were enrolled in the study.

Results: Only $39.7 \%$ of patients with 2 or more risk factors for GI bleeding were prescribed gastroprotective medication at hospital discharge. The probability of elderly antiplatelet drug user for prescription of gastroprotective medication was improved with following risk factors: age $\geq 85$ years (OR $=2.99$ ); history of peptic ulcer disease/ GI bleeding (OR = 15.79); other GI disorders ( $O R=15.48)$; concomitant therapy with drugs increasing the risk of $\mathrm{GI}$ bleeding - systemic corticosteroids $(\mathrm{OR}=29.03)$ and NSAIDs $(\mathrm{OR}=4.79)$.

Conclusion: Results of our study indicate the necessity to increase the awareness of GI bleeding risk in longterm antiplatelet users among prescribing physicians (Tab. 3, Ref. 36). Text in PDF www.elis.sk. Key words: risk factors, gastrointestinal bleeding, peptic ulcer disease, hospitalization, discharge.
\end{abstract}

Antiplatelet agents have become a widely prescribed drug class due to their benefit in secondary prevention of cardiovascular diseases through different age groups. Antiplatelet medication often represents life-long therapy. Dual antiplatelet therapy (combination of aspirin with thienopyridines) plays a key role in cardiovascular prevention in patients with acute coronary syndrome $(1,2)$. Extensive use of antiplatelet agents in clinical practice is associated with the need of prescribers' awareness of adverse effects that may accompany this therapy. The use of acetylsalicylic

${ }^{1}$ Department of Pharmacology and Clinical Pharmacology, Faculty of Medicine, Comenius University in Bratislava, Bratislava, Slovakia, ${ }^{2} 1$ st Department of Internal Medicine, Faculty of Medicine, Comenius University in Bratislava, Bratislava, Slovakia, ${ }^{3}$ Department of Pharmacology and Toxicology, Faculty of Pharmacy, Comenius University in Bratislava, Bratislava, Slovakia, ${ }^{4}$ st Department of Orthopaedics and Traumatology, University Hospital Bratislava, Bratislava, Slovakia, and ${ }^{5}$ Department of Trauma Surgery, University Hospital Bratislava, Bratislava, Slovakia

Address for correspondence: V. Slezakova, Mgr, Department of Pharmacology and Clinical Pharmacology, Faculty of Medicine, Comenius University in Bratislava, Spitalska 24, SK-811 08 Bratislava, Slovakia. Phone: +421.2 .59357229$

Acknowledgment: Our study was supported by grant VEGA 1/0886/14 and VEGA 1/0939/14. The grant providers played no role in the design, methods, data collection, analysis and interpretation of the data, preparation of the paper or in the decision to submit the manuscript. We thank Dr. Agata Argalasova, Dr. Silvia Cervenova and Daniel Klimovsky for their assistance with data collection. acid (ASA) and clopidogrel in monotherapy or in combination is associated with increased risk of gastrointestinal (GI) bleeding (3, 4). The damage of gastric mucosa caused by ASA is mediated by topical and systemic inhibition of cyclooxygenase. The administration of clopidogrel can lead to GI bleeding from preexisting lesions due to reduced platelet aggregation. Clopidogrel also inhibits angiogenesis (e.g. release of vascular endothelial growth factor from platelets). This mechanism negatively influences the process of ulcer healing $(5,6)$. For these reasons, the replacement of ASA with clopidogrel in patients who experienced GI bleeding during antiplatelet therapy does not represent a sufficient tool for prevention of further GI bleeding $(7,8,9)$.

The use of ASA or clopidogrel alone is associated with 1.5 to 2-fold increase of the risk of GI bleeding. Combination of ASA with clopidogrel is related to almost 4-fold increase of this risk (7). Older age, history of GI bleeding, concomitant use of anticoagulants, non-steroidal anti-inflammatory drugs (NSAIDs), corticosteroids and $H$. pylori infection represent the most important risk factors for GI bleeding (10). The relative risk of bleeding in an individual patient increases with increasing number of risk factors. Gastroesophageal reflux disease, dyspepsia, chronic diseases (e.g. heart failure, chronic renal failure, diabetes mellitus) and alcohol abuse might contribute to increased risk of GI bleeding, as well (10).

Elderly patients represent a specific subgroup of antiplatelet users with increased GI bleeding risk. Higher age is accompanied with increased risk of cardiovascular events and at the same time 
with higher risk of GI bleeding. However, several studies reported underuse of preventive therapies in elderly patients $(11,12)$.

The suppression of gastric acid production represents an effective way in prevention of GI complications associated with the use of antiplatelet agents. Proton pump inhibitors (PPIs) produce more intensive and longer suppression of gastric acid production compared to $\mathrm{H}_{2}$-antagonists and are preferred over $\mathrm{H}_{2}$-antagonists in current clinical practice $(4,13,14)$.

Despite beneficial effects of PPIs, there are some controversial issues associated with their use. Concomitant use of thienopyridines and PPIs may lead to drug-drug interactions. Both drugs are metabolized by cytochrome P450 isoenzyme 2C19 (CYP 2C19). Proton pump inhibitors as substrates of CYP 2C19 may decrease metabolic activation of clopidogrel. This interaction may result in decreased antiplatelet effect. Consequently, the risk of thromboembolic events may be increased. Experimental studies support this mechanism of interaction (15, $16,17)$, while evidence from observational studies in human subjects is inconclusive $(18,19,20)$. Nevertheless, in patients who require treatment with clopidogrel the prescription of PPIs without inhibitory effect on CYP 2C19 should be preferred (e.g. pantoprazole).

Although the use of gastroprotective therapy in NSAIDs users was widely described in the literature $(21,22,23,24)$, there are only few studies that assessed the prescription patterns of gastroprotective therapy in patients using antiplatelet agents $(23,25,26)$. For this reason, the aim of our study was to evaluate the use of gastroprotective medication in elderly patients taking antiplatelet drugs in relationship to their GI bleeding risk. We also analyzed the influence of individual patient's risk factors for GI bleeding on the prescription of gastroprotective medication.

\section{Methods}

\section{Study population}

Patients of our retrospective study were hospitalized at departments of Internal medicine in three municipal hospitals in Slovakia (Nitra, Malacky and Ilava) during the period from January 1, 2008 to December 31, 2009. The same source of patients' data was used in previous evaluation (27). Patients who died during hospitalization or those with incomplete medical documentation were excluded from the study. In the study we included only patients who fulfilled following criteria: a) age $\geq 65$ years; b) prescription of low dose ASA ( 75 - $325 \mathrm{mg}$ per day) or clopidogrel (75 mg per day) in monotherapy or combination of both antiplatelet agents at hospital discharge; c) no history of hypersensitivity to PPIs or $\mathrm{H}_{2}$-antagonists.

Basic socio-demographic characteristics (age, gender), smoking status, alcohol abuse and co-morbid conditions were recorded for each patient. Data on diagnoses were collected in compliance with the 10th revision of the International Classification of Diseases (28). For each patient the use of antiplatelet medication at hospital discharge (ASA or clopidogrel in monotherapy or in combination) was recorded. We searched for the prescription of gastroprotective medication (PPIs or $\mathrm{H}_{2}$-antagonists) at hospital discharge.

We divided our study sample into two groups: "gastroprotective medication users" in whom the use of PPIs or $\mathrm{H}_{2}$-antagonists was recorded and "gastroprotective medication non-users" in whom we did not find prescription of PPIs or $\mathrm{H}_{2}$-antagonists. We did not evaluate the prescription of antacids or prostaglandins in our analysis.

Data were collected from patients' medical records. The rules of personal data confidentiality were fully respected.

\section{Risk factors for GI bleeding}

In our analysis, we considered following risk factors for GI bleeding: 1) history of peptic ulcer disease/ GI bleeding; 2) concomitant use of NSAIDs, antithrombotics or systemic corticosteroids (10).

In order to assess the risk factors for GI bleeding in a complex way we evaluated also the presence of other GI disorders such as gastritis, duodenitis, dyspepsia and gastroesophageal reflux disease. We have taken into consideration also factors identified by Moukarbel et al (2009) as independent predictors of GI bleeding in patients taking antiplatelet therapy: male gender, dual antiplatelet therapy, chronic renal failure, heart failure NYHA (New York Heart Association) stage III - IV, diabetes mellitus and alcohol abuse (29).

Patients with only 1 risk factor for GI bleeding (those with no additional risk factors except of age $\geq 65$ years) were considered to be at low risk and patients with 2 or more risk factors to be at high risk of GI bleeding.

We compared the presence of individual risk factors for GI bleeding between the group of "gastroprotective medication users" and "gastroprotective medication non-users".

\section{Statistical analysis}

We evaluated the distribution of categorical variables in the group of "gastroprotective medication users" and "gastroprotective medication non-users" using the $\chi^{2}$ test. In case of small numbers in individual cells of contingency tables $(<5)$ the Fisher exact test was applied. Continuous variables were expressed as mean \pm standard deviation. In order to identify the most significant predictors of gastroprotective medication prescription multivariate analysis namely the binary logistic regression was used. The influence of patients' characteristics on the use of gastroprotective medication was characterized by odds ratio and $95 \%$ confidence interval of odds ratio.

All statistical tests were carried out at the level of significance $\alpha=0.05$. Statistical software IBM SPSS Statistic 20 (IBM SPSS Inc., Chicago, IL, USA) was used.

\section{Results}

Our study population consisted of 428 hospitalized elderly patients. The mean age of study participants was $77.9 \pm 6.5$ years. Women prevailed over men $(255 ; 59.6 \%$ vs $173 ; 40.4 \%$, respectively). Review of medical records revealed GI disorders in 61 
Tab. 1. Univariate analysis of the influence of number of GI bleeding risk factors on the use of gastroprotective medication.

\begin{tabular}{|c|c|c|c|}
\hline $\begin{array}{l}\mathrm{N}^{\circ} \text { of risk factors for } \\
\text { GI bleeding }\end{array}$ & $\begin{array}{c}\text { Gastroprotective } \\
\text { medication } \\
\text { non-users } \\
(\% \text { of } n)\end{array}$ & $\begin{array}{c}\text { Gastroprotective } \\
\text { medication } \\
\text { users } \\
(\% \text { of } n)\end{array}$ & $\mathrm{p}$ \\
\hline $\begin{array}{l}\text { low GI bleeding risk } \\
1(\mathrm{n}=234)\end{array}$ & $188(80.3)$ & $46(19.7)$ & \\
\hline $\begin{array}{l}\text { high GI bleeding risk } \\
\begin{array}{l}2(\mathrm{n}=149) \\
3(\mathrm{n}=41) \\
\geq 4(\mathrm{n}=4)\end{array}\end{array}$ & $\begin{array}{c}102(68.5) \\
15(36.6) \\
0(0.0)\end{array}$ & $\begin{array}{l}47(31.5) \\
26(63.4) \\
4(100.0)\end{array}$ & $\stackrel{0}{\dot{v}}$ \\
\hline
\end{tabular}

Tab. 2 Univariate analysis of the influence of patient's characteristics and GI bleeding risk factors on the prescription of gastroprotective medication at hospital discharge.

\begin{tabular}{|c|c|c|c|}
\hline & $\begin{array}{l}\text { Gastroprotec- } \\
\text { tive medication } \\
\text { non-users } \\
(\mathrm{n}=305)\end{array}$ & $\begin{array}{c}\text { Gastroprotec- } \\
\text { tive medication } \\
\text { users }(\mathrm{n}=123)\end{array}$ & $\mathrm{p}$ \\
\hline \multicolumn{4}{|l|}{ Age } \\
\hline $65-74$ years & $94(30.8)$ & $29(23.6)$ & \\
\hline $75-84$ years & $174(57.0)$ & $63(51.2)$ & \\
\hline$\geq 85$ years & $37(12.1)$ & $31(25.2)$ & 0.003 \\
\hline Male gender & $121(39.7)$ & $52(42.3)$ & 0.619 \\
\hline Alcohol abuse & $37(12.1)$ & $6(4.9)$ & 0.024 \\
\hline \multicolumn{4}{|l|}{ Gastrointestinal disorders } \\
\hline Gastro-oesophageal reflux disease & $3(1.0)$ & $10(8.1)$ & $<0.001^{*}$ \\
\hline Dyspepsia & $6(2.0)$ & $4(3.3)$ & $0.483^{*}$ \\
\hline Gastro- duodenitis & $3(1.0)$ & $8(6.5)$ & $0.003^{*}$ \\
\hline Peptic ulcer disease/GI bleeding & $9(3.0)$ & $18(14.6)$ & $<0.001$ \\
\hline \multicolumn{4}{|l|}{ Co-morbidities } \\
\hline Hypertension & $275(90.2)$ & $106(86.2)$ & 0.233 \\
\hline Heart failure & $91(29.8)$ & $47(38.2)$ & 0.093 \\
\hline NYHA class III - IV & $39(12.8)$ & $15(12.2)$ & 0.867 \\
\hline Atrial fibrillation & $40(13.1)$ & $22(17.9)$ & 0.204 \\
\hline Diabetes mellitus & $142(46.6)$ & $66(53.7)$ & 0.183 \\
\hline Chronic renal insufficiency & $159(52.1)$ & $77(62.6)$ & 0.049 \\
\hline Hepathopathy & $24(7.9)$ & $15(12.2)$ & 0.159 \\
\hline Dementia & $43(14.1)$ & $16(13.0)$ & 0.767 \\
\hline \multicolumn{4}{|l|}{ Established atherosclerotic disease } \\
\hline Myocardial infarction & $71(23.3)$ & $21(17.1)$ & 0.157 \\
\hline Stroke/TIA & $101(33.1)$ & $36(29.3)$ & 0.440 \\
\hline Peripheral artery disease & $25(8.2)$ & $8(6.5)$ & 0.552 \\
\hline \multicolumn{4}{|l|}{ Drugs increasing the risk of GI bleeding } \\
\hline Systemic corticosteroids & $3(1.0)$ & $12(9.8)$ & $<0.001^{*}$ \\
\hline NSAIDs & $14(4.6)$ & $13(10.6)$ & 0.021 \\
\hline Warfarin & $1(0.3)$ & $1(0.8)$ & $0.493^{*}$ \\
\hline LMWH & $26(8.5)$ & $10(8.1)$ & 0.894 \\
\hline \multicolumn{4}{|l|}{ Type of antiplatelet therapy } \\
\hline Clopidogrel in monotherapy & $24(7.9)$ & $12(9.8)$ & \\
\hline ASA in monotherapy & $223(73.1)$ & $93(75.6)$ & \\
\hline Dual antiplatelet therapy & $58(19.0)$ & $18(14.6)$ & 0.498 \\
\hline
\end{tabular}

\begin{tabular}{llll} 
Dual antiplatelet therapy & $58(19.0)$ & $18(14.6)$ & 0.498 \\
\hline$p-$ statistical significance according to the $\chi^{2}$ test $*$ statistical significance according
\end{tabular} to the Fisher's exact test, NYHA - New York Heart Association; TIA - transient ischemic attack; GI - gastrointestinal; NSAIDs - non-steroidal anti-inflammatory drugs; LMWH - low molecular weight heparins; ASA - acetylsalicylic acid

patients (14.3\%), out of which 27 experienced peptic ulcer disease or GI bleeding. The mean number of medications prescribed at hospital discharge was $8.4 \pm 2.9$.

Monotherapy with ASA was the most frequently used antiplatelet medication, prescribed in $316(73.8 \%)$ elderly patients.
Tab. 3 Multivariate analysis of the influence of patient's characteristics and GI bleeding risk factors on the prescription of gastroprotective medication at hospital discharge.

\begin{tabular}{lc}
\hline & OR (CI 95\%) \\
\hline Age $\geq 85$ years & $2.99(1.59-5.64)$ \\
Male gender & $1.23(0.74-2.04)$ \\
Alcohol abuse & $0.35(0.13-0.99)$ \\
Established atherosclerotic disease & $0.89(0.52-1.51)$ \\
Heart failure, NYHA class III - IV & $0.90(0.42-1.96)$ \\
Diabetes mellitus & $1.63(0.99-2.68)$ \\
Chronic renal insufficiency & $0.97(0.58-1.63)$ \\
History of peptic ulcer disease/ GI bleeding & $15.79(3.35-74.31)$ \\
Other GI disorders* & $15.48(3.87-61.94)$ \\
Systemic corticosteroids & $29.03(5.00-168.58)$ \\
NSAIDs & $4.79(1.11-20.70)$ \\
Anticoagulants & $2.00(0.55-7.32)$ \\
Dual antiplatelet therapy & $2.26(0.58-8.75)$ \\
High risk for GI bleeding & $0.47(0.12-1.88)$ \\
\hline
\end{tabular}

"gastritis, duodenitis, dyspepsia and gastroesophageal reflux disease, ${ }^{* *}$ having 2 or more risk factors for GI bleeding, GI - gastrointestinal; NYHA - New York Heart Association; NSAIDs - non-steroidal anti-inflammatory drugs

Prescription of clopidogrel in monotherapy was less common, prescribed in 36 patients $(8.4 \%)$ at hospital discharge. Dual antiplatelet therapy (combination of ASA with clopidogrel) was prescribed in 76 patients $(17.8 \%)$.

We recorded the prescription of $\mathrm{H}_{2}$-antagonists in $60(14.0 \%)$ patients. PPIs were prescribed in 66 patients $(15.4 \%)$.

Factorsinfluencing the prescriptionofgastroprotectivemedication

We identified 234 patients with one risk factor for GI bleeding and classified them as low-risk patients. Patients who had 2 or more risk factors for GI bleeding were considered to be at high risk of GI bleeding $(n=194)$. We revealed underuse of gastroprotective medication in the group of elderly patients with high GI bleeding risk. Only 77 (39.7\%) out of 194 patients at high risk of GI bleeding received PPI or $\mathrm{H}_{2}$-antagonist.

Higher number of risk factors for GI bleeding was associated with increased occurrence of prescription of gastroprotective medication $(p<0.001)$. The relationship between the number of risk factors and the use of gastroprotective medication is shown in Table 1.

The results of univariate analysis of the influence of patients' characteristics on the prescription of gastroprotective medication are shown in Table 2.

The results of multivariate analysis of the influence of evaluated patients' characteristics are shown in Table 3. Patients with history of peptic ulcer disease/ GI bleeding, other GI disorders (gastritis, duodenitis, dyspepsia and gastroesophageal reflux disease), patients aged $\geq 85$ years and those who were prescribed NSAIDs or systemic corticosteroids had increased likelihood of being prescribed gastroprotective medication at hospital discharge. Alcohol abuse was associated with lower chance of a patient to receive gastroprotective medication.

\section{Discussion}

Our study revealed underuse of gastroprotective medication in high-risk antiplatelet users with $\geq 2$ risk factors for GI bleeding. 
Only $39.7 \%$ of these high-risk patients were prescribed gastroprotective medication at hospital discharge. The most important GI bleeding risk factors (history of peptic ulcer disease/ GI bleeding and other GI disorders such as gastritis, duodenitis, dyspepsia and gastroesophageal reflux disease) were associated with increased likelihood of a patient to be prescribed gastroprotective medication at hospital discharge. The oldest group of elderly patients aged $\geq$ 85 years had higher chance of receiving gastroprotective medication, as well. Concomitant therapy with drugs increasing the risk of GI bleeding such as systemic corticosteroids and NSAIDs was also associated with increased likelihood of prescribing the gastroprotective medication. These results indicate that hospital physicians evaluated the necessity of the use of gastroprotective therapy. Particular attention was paid to patients with the presence of individual risk factors mentioned above. Surprisingly, alcohol abuse, which represents another risk factor for GI bleeding in antiplatelet users, decreased the probability for administration of gastroprotective medication.

Similarly to our study, underuse of gastroprotective medication in elderly patients with high risk of GI bleeding was shown in the recent study. De Jong et al (2013) found that prescription of PPIs was missing in $36 \%$ of high-risk patients regularly taking low-dose ASA (26). Similarly, Casado-Arroyo et al (2010) reported underuse of gastroprotective medication among dual antiplatelet therapy users with high risk of GI bleeding in hospital centers in USA and Spain (25). Lanas et al (2011) reported $81 \%$ prevalence of gastroprotective agent prescription in low dose ASA users aged $\geq 65$ years (23). This study showed that the prevalence of the use of gastroprotective medication was higher in low dose ASA users compared to NSAIDs users.

Univariate analysis showed that in our study higher number of risk factors for GI bleeding was associated with increased use of gastroprotective medication. Lanas et al (2011) reported similar findings in NSAIDs users and low dose ASA users (22). However, some studies failed to demonstrate an association between the number of risk factors for GI bleeding and prescription of gastroprotective medication $(24,25)$.

In the oldest group of elderly patients the prescription of preventive therapy is often omitted $(30,31)$. Surprisingly, results of multivariate analysis showed that the oldest patients of our study group ( $\geq 85$ years) had higher probability of receiving gastroprotective medication in comparison with younger elderly ( $<85$ years). Increased prescription of gastroprotective medication in the group of the oldest indicates the awareness of physicians regarding positive association between the risk of GI bleeding and advancing age. Similarly to our study, Medlock et al (2013) found increased prevalence of gastroprotective agents among NSAIDs users in the oldest patients of their study group (32). In contrast to our results, in several studies underuse of gastroprotective medication in the oldest elderly patients was reported $(11,33)$.

In our study, we found that history of peptic ulcer disease/ GI bleeding, and other GI disorders was positively associated with the prescription of gastroprotective medication. These results indicate that GI disorders are well-perceived risk factors for GI bleeding among hospital prescribers. In the study of de Jong et al
(2013) low dose ASA users with history of peptic ulcer disease had 13-fold increased odds of receiving PPIs than those without ulcer disease (26).

Alcohol abuse was shown to predict independently the risk of GI bleeding in patients taking antiplatelet agents (29). However, in our study alcohol abuse was associated with lower chance of a patient to be prescribed gastroprotective medication. The design of our study does not enable to explain possible reasons for underuse of gastroprotective medication in patients with alcohol abuse.

The risk of GI bleeding is significantly increased in patients taking dual antiplatelet therapy compared to those on antiplatelet monotherapy (7). Nevertheless, in our analysis the type of antiplatelet medication (monotherapy or combination) did not influence the distribution of patients between "gastroprotective medication users" and "non-users". On the other hand, antiplatelet therapy users who were concomitantly prescribed NSAIDs or corticosteroids had increased probability of being prescribed gastroprotective medication. According to the results of observational study of Garcia-Rodriguez et al (2011) adding clopidogrel to low dose ASA results in 4-fold increase of the occurrence of GI bleeding events (7). In the same study concomitant use of NSAIDs or corticosteroids with low dose ASA increased the risk of GI bleeding 5- and 8-fold, respectively. Our results suggest that the risk of GI bleeding is generally better perceived by prescribing physicians in NSAIDs and systemic corticosteroid users in comparison with dual antiplatelet therapy users.

Our analysis did not show any difference in the use of gastroprotective medication in patients with established atherosclerotic disease (myocardial infarction, stroke, peripheral arterial disease). The cardiovascular benefits of long-term therapy with low dose ASA are evidence based in patients with established atherosclerotic disease. However, in patients with low cardiovascular risk the benefit of such therapy seems to be outweighed by increased risk of GI complications (34). Recently, de Groot et al (35) published a pharmacoeconomic evaluation of the use of low dose ASA with or without gastroprotective medication in primary and secondary prevention. Primary prevention with low dose ASA was shown to be cost-effective only in combination with PPIs. The possible adverse effects of gastroprotective medication also should be taken into consideration, e.g. bacterial infections of GI tract, pneumonia, magnesium deficiency and increased risk of hip fractures. However, overall incidence of adverse effects mentioned above remains very low. In general PPIs as well as $\mathrm{H}_{2}$-antagonists represent drug classes with favorable safety profile (36).

H. pylori infection represents another well-known risk factor for GI bleeding. This factor was not taken into consideration in our analysis. Reason for this limitation was the lack of relevant data on $H$. pylori infection in the documentation of our study population. However, $H$. pylori infection is often underdiagnosed in clinical practice especially in asymptomatic patients. Therefore we assume that omitting this factor does not influence the relevance of our findings. 


\section{Conclusion}

Our study revealed underutilization of gastroprotective medication in antiplatelet users. However, the most important risk factors for GI bleeding (history of peptic ulcer disease/ GI bleeding, as well as other GI disorders, concomitant use of NSAIDs and systemic corticosteroids) appeared as factors increasing the probability of a patient to be prescribed gastroprotective medication. We consider the finding of increased chance for gastroprotective medication prescription in the oldest patients of our group ( $\geq 85$ years) positive. Hospital physicians are sufficiently aware of the risk of bleeding in case of patients with aforementioned risk factors. However, the overall underuse of gastroprotective medications indicate the necessity to pay more attention to favorable effects of gastroprotective medication in a certain part of antiplatelet drug users during courses of continual medical education.

\section{References}

1. Hamm CW, Bassand JP, Agewall S et al. ESC Committee for Practice Guidelines. ESC Guidelines for the management of acute coronary syndromes in patients presenting without persistent ST-segment elevation: The Task Force for the management of acute coronary syndromes (ACS) in patients presenting without persistent ST-segment elevation of the European Society of Cardiology (ESC). Eur Heart J 2011; 32 (23): 2999 - 3054.

2. Task Force on the management of ST-segment elevation acute myocardial infarction of the European Society of Cardiology (ESC); Steg PG, James SK, Atar D et al. ESC Guidelines for the management of acute myocardial infarction in patients presenting with ST-segment elevation. Eur Heart J 2012; 33 (20): 2569 - 619.

3. Yusuf S, Zhao F, Mehta SR, Chrolavicius S, Tognoni G, Fox KK; Clopidogrel in Unstable Angina to Prevent Recurrent Events Trial Investigators. Effects of clopidogrel in addition to aspirin in patients with acute coronary syndromes without ST-segment elevation. N Engl J Med 2001; 345 (7): $494-502$.

4. Ng FH, Lam KF, Wong SY et al. Upper gastrointestinal bleeding in patients with aspirin and clopidogrel co-therapy. Digestion 2008; 77 (3-4): $173-177$.

5. Luo JC, Huo TI, Hou MC et al. Clopidogrel delays gastric ulcer healing in rats. Eur J Pharmacol 2012; 695 (1-3): 112 - 119.

6. Smadja DM, Bura A, Szymezak J et al. Effect of clopidogrel on circulating biomarkers of angiogenesis and endothelial activation. J Cardiol 2012; 59 (1): $30-35$.

7. Garcia Rodriguez LA, Lin KJ, Hernandez- Diaz S, Johansson S. Risk of upper gastrointestinal bleeding with low-dose acetylsalicylic acid alone and in combination with clopidogrel and other medications. Circulation 2011; 123 (10): 1108 - 1115 .

8. Hsiao FY, Tsai YW, Huang WF et al. A comparison of aspirin and clopidogrel with or without proton pump inhibitors for the secondary prevention of cardiovascular events in patients at high risk for gastrointestinal bleeding. Clin Ther 2009; 31 (9): 2038 - 47.

9. Chan FK, Ching JY, Hung LC et al. Clopidogrel versus aspirin and esomeprazole to prevent recurrent ulcer bleeding. N Engl J Med 2005; 352 (3): $238-244$.
10. Bhatt DL, Scheiman J, Abraham NS et al. American College of Cardiology Foundation; American College of Gastroenterology; American Heart Association. ACCF/ACG/AHA 2008 expert consensus document on reducing the gastrointestinal risks of antiplatelet therapy and NSAID use. Am J Gastroenterol 2008; 103 (11): 2890 - 2907.

11. Barry PJ, Gallagher P, Ryan C, O‘mahony D. START (screening tool to alert doctors to the right treatment)--an evidence-based screening tool to detect prescribing omissions in elderly patients. Age Ageing 2007; 36 (6): $632-638$.

12. Spinewine A, Schmader KE, Barber $\mathbf{N}$ et al. Appropriate prescribing in elderly people: how well can it be measured and optimised? Lancet 2007; 370 (9582): 173 - 184.

13. Ray WA, Murray KT, Griffin MR et al. Outcomes with concurrent use of clopidogrel and proton-pump inhibitors: a cohort study. Ann Intern Med 2010; 152 (6): 337 - 145.

14. Ng FH, Wong SY, Lam KF et al. Famotidine is inferior to pantoprazole in preventing recurrence of aspirin-related peptic ulcers or erosions. Gastroenterology 2010; 138 (1): $82-88$.

15. Roden DM, Stein CM. Clopidogrel and the concept of high-risk pharmacokinetics. Circulation 2009; 119 (16): 2127 - 2130.

16. Cuisset T, Frere C, Quilici J et al. Comparison of omeprazole and pantoprazole influence on a high 150-mg clopidogrel maintenance dose the PACA (Proton Pump Inhibitors And Clopidogrel Association) prospective randomized study. J Am Coll Cardiol 2009; 54 (13): 1149 - 1153 .

17. Siller-Matula JM, Spiel AO, Lang IM, Kreiner G, Christ G, Jilma B. Effects of pantoprazole and esomeprazole on platelet inhibition by clopidogrel. Am Heart J 2009; 157 (1): 148.

18. O`Donoghue ML, Braunwald E, Antman EM et al. Pharmacodynamic effect and clinical efficacy of clopidogrel and prasugrel with or without a proton-pump inhibitor: an analysis of two randomised trials. Lancet 2009; 374 (9694): 989 - 997.

19. Dunn SP, Macaulay TE, Brennan DM. Baseline proton pump inhibitor use is associated with increased cardiovascular events with and without use of clopidogrel in the CREDO trial. Circulation 2008; 118 : S815 Abstract

20. Bhatt DL, Cryer BL, Contant CF et al. COGENT Investigators. Clopidogrel with or without omeprazole in coronary artery disease. $\mathrm{N}$ Engl J Med 2010; 363 (20): 1909 - 1917.

21. Sturkenboom MC, Burke TA, Dieleman JP, Tangelder MJ, Lee F, Goldstein JL. Underutilization of preventive strategies in patients receiving NSAIDs. Rheumatology (Oxford) 2003; 42 Suppl 3: 23 - 31.

22. Thiéfin G, Schwalm MS. Underutilization of gastroprotective drugs in patients receiving non-steroidal anti-inflammatory drugs. Dig Liver Dis 2011; 43 (3): 209 - 214.

23. Lanas A, Plazas MA, Gimeno E, Muñoz-Tudurí M. Gastroprotection in NSAID and low-dose aspirin users: a cross-sectional study in primary care. Gastroenterol Hepatol 2012; 35 (1): 1 - 7.

24. Carvajal A, Arias LH, Vega E et al. Gastroprotection during the administration of non steroidal anti-inflammatory drugs. A drug-utilization study. Eur J Clin Pharmacol 2004; 60 (6): 439 - 444.

25. Casado-Arroyo R, Scheiman JM, Polo-Tomas M et al. Underutilization of gastroprotection for at-risk patients undergoing percutaneous coronary intervention: Spain compared with the United States. Aliment Pharmacol Ther 2010; 32 (5): $689-695$. 
643-648

26. De Jong HJ, Korevaar JC, van Dijk L, Voogd E, van Dijk CE, van Oijen MG. Suboptimal prescribing of proton-pump inhibitors in lowdose aspirin users: a cohort study in primary care. BMJ Open 2013; 3 (7).

27. Wawruch M, Macugova A, Kostkova L et al. The use of medications with anticholinergic properties and risk factors for their use in hospitalised elderly patients. Pharmacoepidemiol Drug Saf 2012; 21 (2): 170 - 176.

28. http://apps.who.int/classifications/icd10/browse/2010/en

29. Moukarbel GV, Signorovitch JE, Pfeffer MA et al. Gastrointestinal bleeding in high risk survivors of myocardial infarction: the VALIANT Trial. Eur Heart J 2009; 30 (18): 2226 - 2232.

30. Kuzuya M, Masuda Y, Hirakawa $Y$ et al. Underuse of medications for chronic diseases in the oldest of community-dwelling older frail Japanese. J Am Geriatr Soc 2006; 54 (4): 598 - 605.

31. Gallagher PF, O`Connor MN, O`Mahony D. Prevention of potentially inappropriate prescribing for elderly patients: a randomized controlled trial using STOPP/START criteria. Clin Pharmacol Ther 2011; 89 (6): $845-854$.

32. Medlock S, Eslami S, Askari M et al. Co-prescription of gastroprotective agents and their efficacy in elderly patients taking nonsteroidal anti-inflammatory drugs: a systematic review of observational studies. Clin Gastroenterol Hepatol. 2013; 11 (10): 1259 - 1269.

33. Morini S, Zullo A, Oliveti D et al. A very high rate of inappropriate use of gastroprotection for nonsteroidal anti-inflammatory drug therapy in primary care: a cross-sectional study. J Clin Gastroenterol 2011; 45 (9): $780-784$.

34. Antithrombotic Trialists‘(ATT) Collaboration. Aspirin in the primary and secondary prevention of vascular disease: collaborative metaanalysis of individual participant data from randomised trials. Lancet 2009; 373 (9678): 1849 - 1860 .

35. De Groot NL, van Haalen HG, Spiegel BM et al. Gastroprotection in Low-Dose Aspirin Users for Primary and Secondary Prevention of ACS: Results of a Cost-Effectiveness Analysis Including Compliance. Cardiovasc Drugs Ther 2013; 27 (4): 341 - 357.

36. Martin RM, Dunn NR, Freemantle S, Shakir S. The rates of common adverse events reported during treatment with proton pump inhibitors used in general practice in England: cohort studies. Br J Clin Pharmacol 2000; 50 (4): $366-372$.

Received January 14, 2014. Accepted May 18, 2014. 